\title{
SEMIALGEBRAICITY OF CERTAIN LOCAL LOCI
}

\author{
F. ACQUISTAPACE \\ Dipartimento di Matematica, Università di Pisa \\ Via Buonarroti 2, 56127 Pisa, Italy \\ E-mail: acquistf@gauss.dm.unipi.it \\ C. ANDRADAS \\ Departamento de Algebra, Facultad de Matemáticas, Universidad Complutense \\ 28040 Madrid, Spain \\ E-mail: andradas@sunal1.mat.ucm.es \\ F. BROGLIA \\ Dipartimento di Matematica, Università di Pisa \\ Via Buonarroti 2, 56127 Pisa, Italy \\ E-mail: broglia@gauss.dm.unipi.it
}

To Prof. S. Eojasiewicz on his 70th birthday

Introduction. Let $A$ and $B$ be two semialgebraic subsets of a compact algebraic variety $M \subset \mathbb{R}^{n}$. In studying when $A$ and $B$ can be separated by a polynomial, cf. $[\mathrm{AcAnBg}]$, one sees that different kind of obstructions can appear. Some are of local nature in the sense that they are concentrated at a point, like in Figure 1 below, where $A$ and $B$ cannot be separated in any neighbourhood of the origin, while some others are not, like in Figure 2, where $A$ and $B$ can be separated locally at all its points although they are not globally separable.

Similar considerations can be made in studying when an open semialgebraic subset $S \subset M$ is basic or not. Thus, it is natural to consider the set $\mathcal{L}_{\mathcal{P}}$ of points $p \in M$ where a certain property $\mathcal{P}$ holds locally, in the sense that it holds for any neighbourhood $U$ of $p$, and ask whether $\mathcal{L}_{\mathcal{P}}$ is semialgebraic. In particular we consider the sets $\mathcal{S}(A, B)$ of

First and third authors are members of GNSAGA of CNR, and partially supported by MURST. Second author partially supported by DGES PB95-0354. All authors partially supported by the EC contract CHRX-CT94-0506.

1991 Mathematics Subject Classification: Primary 14Pxx.

Received by the editors: December 20, 1996; in the revised form: August 13, 1997.

The paper is in final form and no version of it will be published elsewhere. 
points $p \in M$ such that $A$ and $B$ cannot be separated in any neighbourhood of $p$ and the set $\mathcal{B}(S)$ of points $p \in M$ such that $S$ is generically basic for any neighbourhood $U$ of $p$. Note that Figure 2 shows that $\mathcal{S}(A, B)(\operatorname{resp} . \mathcal{B}(A))$ may be empty, even if $A$ and $B$ are not separable (resp. $S$ is not basic) in $M$. Also note that neither of the properties defining $\mathcal{S}(A, B)$ or $\mathcal{B}(S)$ can be described by a first order formula, so that the question is not obvious. In this note we show that over the real numbers (i.e., $M \subset \mathbb{R}^{n}$ ), they are semialgebraic. Indeed, we obtain our result as a consequence of the separation criterion obtained in [AcAnBg, Theorem 4.5] and the basicness criterion of [AcBgVe, Theorem 4.1], which hold only for the real numbers. We also include an example which shows that the sets $\mathcal{S}(A, B), \mathcal{B}(S)$, need not be semialgebraic when we work over a non-archimedean real closed field.

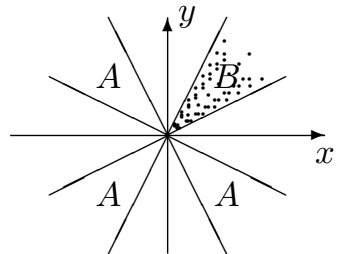

Figure 1

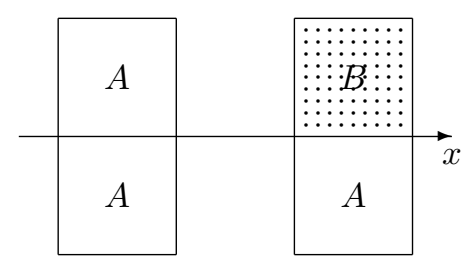

Figure 2

Here is how we proceed. We work out completely the solution for the separation problem, and sketch only the answer for the basicness question in Section 5, which is completely analogous. As it is customary, we deal first with the generic problem, i.e., we consider the set $\mathcal{S}_{\text {gen }}(A, B)$ of points $p \in M$ such that $A$ and $B$ cannot be generically separated in any neighbourhood of $p$, and we show, using the separation criterion quoted above, that $\mathcal{S}_{\text {gen }}(A, B)$ is semialgebraic. Since the separation criterion holds only under special assumptions, namely that $M$ is non-singular and the walls of $A$ and $B$ are nonsingular and normal crossings, we consider first this situation. Then, to pass to the general case, we study the behaviour of the set $\mathcal{S}_{\text {gen }}(A, B)$ under the desingularization map $\pi: M^{\nu} \rightarrow M$. To this respect we point out that, in going upstairs from $M$ to $M^{\nu}$ by $\pi$, we may miss some points of $\mathcal{S}_{\text {gen }}(A, B)$, as it happens in the examples of the pictures above, where Figure 2 is the blow-up of Figure 1 to place the walls of $A$ and $B$ in normal crossings. In fact, what happens is that $p$ lies in $\mathcal{S}_{\text {gen }}(A, B)$ if and only if $\pi^{-1}(A)$ and $\pi^{-1}(B)$ are not separable in a neighbourhood of $\pi^{-1}(p)$. Thus we have to deal with the notion of $A$ and $B$ to be generically separable in a neighbourhood of a subset $Z \subset M^{\nu}$. We characterize when this is possible in combinatorial terms by means of what we call the separation tree of $A$ and $B$ (cf. Proposition 2.1 below). Next we stratify $M^{\nu}$ and the map $\pi$ in such a way that the separation tree is compatible with the stratification, and we are able to descend the results from $M^{\nu}$ to $M$. Finally we write $\mathcal{S}(A, B)$ as a finite union of $\mathcal{S}_{\text {gen }}(C, D)$ for certain $C$ and $D$, which shows that it is semialgebraic.

We want to thank M. P. Vélez for many fruitful conversations. In particular the idea of building a separation tree was inspired by her. The example of the last section of the paper was built during the Oberwolfach meeting on real geometry in March 1997, in conversations with L. Bröcker, M. Coste and A. Prestel. Finally, we thank the Universities of Pisa and Complutense de Madrid for partially supporting this research by means of their bilateral agreement. 
1. The case of normal crossings. We start by fixing the notation. Let $M \subset \mathbb{R}^{n}$ be a compact irreducible algebraic variety. We say that two semialgebraic subsets of $M$ are generically equal if and only if they coincide up to a subset of codimension at least 1 . In particular, for any semialgebraic subset $S \subset M$ we set $S^{*}=\operatorname{Int}_{M} \operatorname{Adh}_{M} S$, where the interior and closure are taken in $M$ with the euclidean topology induced by $\mathbb{R}^{n}$. We will drop the subindex $M$ whenever no confusion is possible. Note that $S^{*}$ is the largest open semialgebraic set which is generically equal to $S$, and an immediate computation shows that $A^{*} \cap B^{*}=(A \cap B)^{*}$. We denote by $\mathcal{R}(M)$ the ring of regular functions on $M$ and by $\Sigma_{M}$ the space of orderings of its field of fractions, cf. [AnBrRz] for the definition and basic properties. For any semialgebraic set $S \subset M$ we consider its tilde $\widetilde{S}$ as the set of orderings of $\Sigma_{M}$ verifying the same sign conditions that define $S$. We just recall that $A$ and $B$ have the same tilde image in $\Sigma_{M}$ if and only if they are generically equal. The walls of $S$ in $M$ are defined as the codimension 1 irreducible components of the Zariski closure, $\overline{\partial S}^{Z}$, of the boundary of $S$.

Now, let $A$ and $B$ be semialgebraic subsets of $M$. We say that $A$ and $B$ are separable if there exists a regular function $f \in \mathcal{R}(M)$ such that $f(A)>0$ and $f(B)<0$. We say that $A$ and $B$ are generically separable if there exists a non-zero regular function $f \in \mathcal{R}(M)$ such that $f(A) \geq 0$ and $f(B) \leq 0$, or equivalently, $A$ and $B$ are generically separable if there exist a proper algebraic subset $Y \subset M$ such that $A \backslash Y$ and $B \backslash Y$ are separable.

We shall say that the triple $(M, A, B)$ verifies the condition (NC) (for Normal Crossings), or simply that it is (NC), if $M$ is non-singular and the walls of $A$ and $B$ are also non-singular and normal crossings. For any subvariety $W \subset M$ we define the shadows $A_{W}, B_{W}$ of $A$ and $B$ in $W$ as $A_{W}=\bar{A} \cap W$ and $B_{W}=\bar{B} \cap W$. Moreover, if $(M, A, B)$ is (NC) and $W$ is a codimension 1 subvariety, we define the counter-shadows $A_{W}^{\prime}, B_{W}^{\prime}$ of $A$ and $B$ in $W$ as follows: let $t \in \mathcal{R}(M)$ be a generator of $\mathcal{J}(W)$ in $\mathcal{R}(M)_{\mathcal{J}(W)}$ and set

$$
\begin{aligned}
& A^{\prime}=(A \cap\{t>0\}) \cup(B \cap\{t<0\}), \\
& B^{\prime}=(A \cap\{t<0\}) \cup(B \cap\{t>0\}) .
\end{aligned}
$$

Then the counter-shadows are the shadows in $W$ of the sets $A^{\prime}$ and $B^{\prime}$. Obviously counter-shadows depend on the choice of $t$. However, in what concerns the separation of $A$ and $B$ "around" $W$ the choice of $t$ is irrelevant. In fact, we have

Theorem 1.1 (Separation criterion [AcAnBg, Theorem 4.5]). Assume that $(M, A, B)$ is (NC). Then $A$ and $B$ are generically separable if and only if for any wall $W$ of $A$ and $B$ in $M$, either the shadows or the counter-shadows (with respect to any choice of $t$ ) of $A$ and $B$ are generically separable in $W$.

For our purposes, we will choose $t$ in such a way that allows induction in the proof of Theorem 1.6 below. More precisely, notice that, in principle, we can take as $t \in \mathcal{R}(M)$ any generator of the ideal $\mathcal{J}\left(W \cup W^{\prime}\right)$, where $W^{\prime}$ is an algebraic variety different from $W$ ) with $\left[W \cup W^{\prime}\right]=0$ in $H_{d-1}\left(M, \mathbb{Z}_{2}\right)$, that is, with $[W]=\left[W^{\prime}\right]$, cf. [BCR, Proposition 12.4.6]. Now, since $[W] \in H_{d-1}^{\text {alg }}\left(M, \mathbb{Z}_{2}\right)$ and is non-singular, we can take $W^{\prime}$ non-singular and normal crossing with $\overline{\partial A}^{Z} \cup \overline{\partial B}^{Z}$, cf. [BCR, Theorem 12.4.10]. Thus, we may take $t$ such that its zero set is at normal crossings with the other walls of $A$ and $B$. From now on, we assume that whenever counter-shadows of two sets are constructed, they are taken with respect to a generator $t$ such that its zero set is at normal crossings with the walls of the sets. 
Since $M$ is compact, any ordering $\alpha \in \Sigma_{M}$ specializes to a point $p \in M$. Now, given $p \in M$ we denote by $\mathcal{G}_{p}$ the set of generizations of $p$, i.e.,

$$
\mathcal{G}_{p}=\left\{\alpha \in \Sigma_{M} \mid \alpha \rightarrow p\right\}=\bigcap_{f}\{f>0\}
$$

where the intersection runs over all functions $f \in \mathcal{R}(M)$ with $f(p)>0$. Thus, $\mathcal{G}_{p}$ is a proconstructible set of $\Sigma_{M}$ and in particular is a subspace of it, cf. [AnBrRz, IV.1.2]. More generally, given a closed semialgebraic subset $Z \subset M$, we define the set

$$
\mathcal{G}_{Z}=\bigcup_{p \in Z} \mathcal{G}_{p}=\left\{\alpha \in \Sigma_{M} \mid \alpha \rightarrow p \in Z\right\}=\bigcap_{f}\{f>0\}
$$

where now the intersection runs over all functions $f \in \mathcal{R}(M)$ with $f(p)>0$ for all $p \in Z$. It follows that $\mathcal{G}_{Z}$ is also a subspace of $\Sigma_{M}$.

Remark 1.2. Notice that in the particular case when $Z$ is a subvariety $W$ of codimension 1 , the space $\mathcal{G}_{W}$ is different from the space $\Sigma_{W}\left[\mathbb{Z}_{2}\right]$ considered in $[\mathrm{AcAnBg}]$. The latter consists only of the orderings of $\Sigma_{M}$ which specializes to some order of $\Sigma_{W}$, and therefore we have $\Sigma_{W}\left[\mathbb{Z}_{2}\right] \subset \mathcal{G}_{W}$. More generally, for any closed semialgebraic set $Z$ and any codimension 1 subvariety $W$, we have

$$
\Sigma_{W}\left[\mathbb{Z}_{2}\right] \cap \mathcal{G}_{Z} \subset \mathcal{G}_{Z \cap W} \subset \mathcal{G}_{Z}
$$

which in particular implies that $\Sigma_{W}\left[\mathbb{Z}_{2}\right] \cap \mathcal{G}_{Z}=\emptyset$ whenever $W \cap Z=\emptyset$.

Geometrically, $\Sigma_{W}\left[\mathbb{Z}_{2}\right]$ and $\mathcal{G}_{W}$ have also different meanings: while separation of $\widetilde{A}$ and $\widetilde{B}$ in $\Sigma_{W}\left[\mathbb{Z}_{2}\right]$ yields to the generic separation of $A$ and $B$ in a "sausage-like open neighbourhood" $U$ of $W$ (that is, $U$ is a neighbourhood only of $W \backslash Y$, where $Y$ is proper subvariety of $W$ ), separation of $\widetilde{A}$ and $\widetilde{B}$ in $\mathcal{G}_{W}$ yields to the generic separation of $A$ and $B$ in a true neighbourhood of $W$. In fact, we have

LEMMA 1.3. $A$ and $B$ are generically separable in a neighbourhood of $Z$ if and only if $\widetilde{A}$ and $\widetilde{B}$ are separable in $\mathcal{G}_{Z}$.

Proof. Suppose that $A$ and $B$ are separable in a neighbourhood $U$ of $Z$. Then $\widetilde{A \cap U}=\widetilde{A} \cap \widetilde{U}$ and $\widetilde{B \cap U}=\widetilde{B} \cap \widetilde{U}$ are separable in $\Sigma_{M}$. Since $\mathcal{G}_{Z} \subset \widetilde{U}$ it follows that $\widetilde{A}$ and $\widetilde{B}$ are separable in $\mathcal{G}_{Z}$.

Conversely, assume that $\widetilde{A}$ and $\widetilde{B}$ are separable in $\mathcal{G}_{Z}$, say $\widetilde{A} \cap \mathcal{G}_{Z} \subset\{g>0\}$ and $\widetilde{B} \cap \mathcal{G}_{Z} \subset\{g<0\}$. Then we have

$$
\begin{aligned}
& \widetilde{A} \cap \bigcap_{f}\{f>0\} \cap\{g<0\}=\emptyset, \\
& \widetilde{B} \cap \bigcap_{f}\{f>0\} \cap\{g>0\}=\emptyset,
\end{aligned}
$$

where the big intersection runs over all functions $f \in \mathcal{R}(M)$ with $\left.f\right|_{Z}>0$. By compactness of $\Sigma_{M}$, there is a finite intersection $U=\left\{f_{1}>0, \ldots, f_{r}>0\right\}$ such that

$$
\begin{aligned}
& \widetilde{A} \cap U \cap\{g<0\}=\emptyset, \\
& \widetilde{B} \cap U \cap\{g>0\}=\emptyset,
\end{aligned}
$$

which means that $A$ and $B$ are generically separated in the neighbourhood $U$ of $Z$. 
Remark 1.4. The link between separation in $\mathcal{G}_{Z}$ and separation in the subspaces $\Sigma_{W}\left[\mathbb{Z}_{2}\right]$ comes as in [AcAnBg]. Taking as ambient space $\mathcal{G}_{Z}$ instead of $\Sigma_{M}$ and arguing exactly as in the proof of $[\mathrm{AcAnBg}$, Proposition 3.3] we get that $\widetilde{A}$ and $\widetilde{B}$ are separable in $\mathcal{G}_{Z}$ if and only if they are so in $\Sigma_{W}\left[\mathbb{Z}_{2}\right] \cap \mathcal{G}_{Z}$ for all walls $W$ in some model of $M$, or equivalently for all walls intersecting the image of $Z$ in some model of $M$. Now, if $(M, A, B)$ is (NC) then the same proof as in $[\mathrm{AcAnBg}$, Theorem 4.1] shows that we need to check this property only for the walls $W$ of $A$ and $B$ in $M$.

This remark yields:

Corollary 1.5. Assume that $(M, A, B)$ is (NC). Then $A$ and $B$ are generically separable in a neighbourhood of $Z$ if and only if $Z \cap\left(A^{*} \cap B^{*}\right)=\emptyset$ and for all walls $W$ of $A$ and $B$ with $Z \cap W \neq \emptyset$ either the shadows $A_{W}, B_{W}$ or the counter-shadows $A_{W}^{\prime}, B_{W}^{\prime}$ are generically separable in a neighbourhood of $Z \cap W$ in $W$.

Pr o of. By Lemma 1.3, $A$ and $B$ are generically separable in a neighbourhood of $Z$ if and only if $\widetilde{A}$ and $\widetilde{B}$ are separable in $\mathcal{G}_{Z}$. Now, the condition $Z \cap\left(A^{*} \cap B^{*}\right)=\emptyset$ is equivalent to $\widetilde{A}$ and $\widetilde{B}$ be disjoint in $\mathcal{G}_{Z}$, and the previous remark shows that $\widetilde{A}$ and $\widetilde{B}$ are separable in $\mathcal{G}_{Z}$ if and only if they are so in all $\Sigma_{W}\left[\mathbb{Z}_{2}\right]$ for the walls $W$ with $W \cap Z=\emptyset$. Then the result follows at once from [AcAnBg, Corollary 4.4] (cf. also [AcAnBg, Theorem 4.5]).

Theorem 1.6. Assume that $(M, A, B)$ is (NC). Then the set $\mathcal{S}_{\text {gen }}(A, B)$ of points of non-local generic separation is closed semialgebraic.

Proof. It is immediate that $\mathcal{S}_{\text {gen }}(A, B)$ is closed. To see that it is semialgebraic we work by induction on $d=\operatorname{dim} M$. If $d=1$ then $A$ and $B$ are generically separated if and only if $A^{*} \cap B^{*}=\emptyset$. So, $\mathcal{S}_{\text {gen }}(A, B)=\overline{A^{*} \cap B^{*}}$, which is semialgebraic.

Assume now that the statement holds for any variety of dimension less than $d$ and set $S_{0}=A^{*} \cap B^{*}$. For any wall $W$ of $A$ and $B$, the triples $\left(A_{W}, B_{W}, W\right)$ and $\left(A_{W}^{\prime}, B_{W}^{\prime}, W\right)$ of the shadows and counter-shadows are again (NC) (remember that we are chosing the parameter $t$ so that this holds). Thus, we may apply induction and get that $\mathcal{S}_{\text {gen }}\left(A_{W}, B_{W}\right)$ and $\mathcal{S}_{\text {gen }}\left(A_{W}^{\prime}, B_{W}^{\prime}\right)$ are semialgebraic. We claim that

$$
\mathcal{S}_{\text {gen }}(A, B)=\overline{S_{0}} \cup\left(\bigcup_{W}\left(\mathcal{S}_{\text {gen }}\left(A_{W}, B_{W}\right) \cap \mathcal{S}_{\text {gen }}\left(A_{W}^{\prime}, B_{W}^{\prime}\right)\right)\right)
$$

Indeed, obviously $S_{0} \subset \mathcal{S}_{\text {gen }}(A, B)$, and since this set is closed we get $\overline{S_{0}} \subset \mathcal{S}_{\text {gen }}(A, B)$. Now, let $p \in \mathcal{S}_{\text {gen }}(A, B) \backslash \overline{S_{0}}$. Then, by Corollary 1.5 there is a wall $W$, with $p \in W$ such that both couples, $A_{W}$ and $B_{W}$, and $A_{W}^{\prime}$ and $B_{W}^{\prime}$ are not generically separated in any neighbourhood of $p$, that is, $p \in \mathcal{S}_{\text {gen }}\left(A_{W}, B_{W}\right) \cap \mathcal{S}_{\text {gen }}\left(A_{W}^{\prime}, B_{W}^{\prime}\right)$.

2. The separation tree. Corollary 1.5 suggests a recursive method to check whether $A$ and $B$ are generically separable in a neighbourhood of $Z$ : one has to consider the walls, check there the property for the shadows and counter-shadows and so on. In this section we formalize this procedure in what we call the separation tree, $\mathcal{T}_{A, B}$, for $A$ and $B$, whose nodes will be triples $(N, C, D)$ verifying the condition (NC), and which is constructed recursively as follows: 
1. We start with the single node $(M, A, B)$.

2. For each node $(N, C, D)$ with $\operatorname{dim} N>1$ consider $C^{\mathrm{b}}=C \backslash(C \cap D)^{*}$ and $D^{\mathrm{b}}=$ $D \backslash(C \cap D)^{*}$. For each wall $W$ of $C^{b}$ and $D^{\mathrm{b}}$ we define two edges descending from $(N, C, D)$ and ending respectively in the triples $\left(W,\left(C^{b}\right)_{W},\left(D^{b}\right)_{W}\right)$ and $\left(W,\left(C^{b}\right)_{W}^{\prime},\left(D^{b}\right)_{W}^{\prime}\right)$ produced by the shadows and counter-shadows of $C^{b}$ and $D^{b}$ in $W$.

Note that for any node $(N, C, D)$, the subtree of $\mathcal{T}_{A, B}$ "dominated" by $(N, C, D)$ is precisely the separation tree $\mathcal{T}_{C, D}$ of $C$ and $D$. Also, we call a complete binary subtree of $\mathcal{T}_{A, B}$ any binary subtree starting at $(M, A, B)$ and such that for each node $(N, C, D)$ the two edges descending from it correspond to the same wall (and therefore consists of the shadows and counter-shadows of $C^{b}$ and $D^{b}$ in it).

We have

Proposition 2.1. Let $(M, A, B)$ be (NC) and let $Z$ be a closed semialgebraic subset of $M$. Then $A$ and $B$ are generically separated in a neighbourhood of $Z$ if and only if for any complete binary subtree of $\mathcal{T}_{A, B}$ there exists a branch of maximal height such that all its nodes $(N, C, D)$ verify $Z \cap(C \cap D)^{*}=\emptyset$.

Pro of. We work by induction on the height of the tree. If $\mathcal{T}_{A, B}$ has height one the statement is trivial. Now, suppose that $A$ and $B$ are generically separated in a neighbourhood of $Z$. Then, by Corollary 1.5, $Z \cap(A \cap B)^{*}=\emptyset$ and for any wall $W$ with $W \cap Z \neq \emptyset$ either $A_{W}, B_{W}$ or $A_{W}^{\prime}, B_{W}^{\prime}$ are generically separated in a neighbourhood of $Z \cap W$. Suppose that it is the former. Now the separation tree $\mathcal{T}_{A_{W}, B_{W}}$ of $A_{W}$ and $B_{W}$ has height less than the separation tree $\mathcal{T}_{A, B}$ of $A$ and $B$, so that by the induction hypothesis there is a branch of maximal height such that all its nodes $(N, C, D)$ verify $(Z \cap W) \cap(C \cap D)^{*}=\emptyset$. Adding the node $(M, A, B)$ above this branch (which starts at $\left.\left(W, A_{W}, B_{W}\right)\right)$ we are done.

Conversely, if $A$ and $B$ are not generically separated in any neighbourhood of $Z$, by Corollary 1.5, either $Z \cap(A \cap B)^{*}=\emptyset$ or there is a wall $W$ with $Z \cap W \neq \emptyset$, in which both couples $A_{W}, B_{W}$ and $A_{W}^{\prime}, B_{W}^{\prime}$ are not generically separated in any neighbourhood of $Z \cap W$. By induction, in both separation trees $\mathcal{T}_{A_{W}, B_{W}}$ and $\mathcal{T}_{A_{W}^{\prime}, B_{W}^{\prime}}$ of $A_{W}, B_{W}$ and $A_{W}^{\prime}, B_{W}^{\prime}$ we find a complete binary subtree such that in all its branches of maximal length there is a node $(N, C, D)$ with $Z \cap(C \cap D)^{*} \neq \emptyset$. Joining these two subtrees with the node $(M, A, B)$ we get a complete binary subtree of $\mathcal{T}_{A, B}$ in which the condition of the statement fails.

3. The general case. Assume now that $(M, A, B)$ is arbitrary. Then by Hironaka's desingularization Theorems I and II, [Hk], there is a regular map $\pi: M^{\nu} \rightarrow M$ such that

1. $M^{\nu}$ is a non-singular variety.

2. $\pi$ is a finite sequence of blowings-up along non-singular algebraic centres so that there is a proper algebraic subset $Y \subset M$ such that

$$
\left.\pi\right|_{M^{\nu} \backslash \pi^{-1}(Y)}: M^{\nu} \backslash \pi^{-1}(Y) \rightarrow M \backslash Y
$$

is a biregular isomorphism.

3. $\pi$ is surjective over the points of $M$ of maximal dimension.

4. The walls of the strict transforms $A^{\nu}, B^{\nu}$, of $A$ and $B$ are non-singular and normal crossings. 
Let $W_{1}, \ldots, W_{r}$ be the walls of $A^{\nu}$ and $B^{\nu}$ in $M^{\nu}$. We consider the stratification $\mathcal{S}=\left\{S_{k}\right\}$ of $M^{\nu}$ induced by these walls as follows. Define

$$
\begin{aligned}
X^{(0)} & =M, \\
X^{(1)} & =\bigcup_{i} W_{i}, \\
X^{(2)} & =\bigcup_{i \neq j}\left(W_{i} \cap W_{j}\right), \\
& \cdots \\
X^{(h)} & =\bigcup_{i_{1} \ldots i_{h}}\left(W_{i_{1}} \cap \ldots \cap W_{i_{h}}\right),
\end{aligned}
$$

where all indices $i_{1} \ldots i_{h}$ are different. Then take as strata $\left\{S_{k}\right\}$ the connected components of the differences $X^{(h)} \backslash X^{(h+1)}$. Note that by construction, for each node $(N, C, D)$ in the separation tree $\mathcal{T}_{A^{\nu}, B^{\nu}}$ of $A^{\nu}$ and $B^{\nu}$, the set $(C \cap D)^{*}$ is a union of strata.

Finally, by [BCR, Theorem 9.3.1], we find a finite semialgebraic partition $M=\bigcup_{i=1}^{r} T_{i}$ and for any $i$ a semialgebraic set $F_{i}$, semialgebraic subsets $\left\{F_{i j}\right\}_{j=1, \ldots, q}$ of $F_{i}$ and a semialgebraic homeomorphism $\theta_{i}: T_{i} \times F_{i} \rightarrow \pi^{-1}\left(T_{i}\right)$ such that $\pi \circ \theta_{i}: T_{i} \times F_{i} \rightarrow T_{i}$ is the projection and $\theta_{i}\left(T_{i} \times F_{i j}\right)=S_{j} \cap \pi^{-1}\left(T_{i}\right)$. Obviously, $A$ and $B$ are generically separated in a neighbourhood of a point $p \in M$ if and only if $A^{\nu}$ and $B^{\nu}$ are generically separated in a neighbourhood of $\pi^{-1}(p)$. Now, using Proposition 2.1, we get

Theorem 3.1. The set $\mathcal{S}_{\text {gen }}(A, B)$ is a union of strata $T_{i}$. In particular $\mathcal{S}_{\text {gen }}(A, B)$ is a (closed) semialgebraic set.

Proof. We have to see that if $y_{1}, y_{2} \in T_{i}$ then $A$ and $B$ are generically separated in a neighbourhood of $y_{1}$ if and only if they are so in a neighbourhood of $y_{2}$, or equivalently, that $A^{\nu}$ and $B^{\nu}$ are generically separated in a neighbourhood of $\pi^{-1}\left(y_{1}\right)$ if and only if they are so in a neighbourhood of $\pi^{-1}\left(y_{2}\right)$. By Proposition 2.1 it is enough to verify that for a given node $(N, C, D)$ in the separation tree $\mathcal{T}_{A^{\nu}, B^{\nu}}, \pi^{-1}\left(y_{1}\right) \cap(C \cap D)^{*} \neq \emptyset$ if and only if $\pi^{-1}\left(y_{2}\right) \cap(C \cap D)^{*} \neq \emptyset$. Since by construction fibres over points in the same $T_{i}$ meet the same strata $S_{k}$, and $(C \cap D)^{*}$ is union of strata, this follows at once.

4. Semialgebraicity of $\mathcal{S}(A, B)$. We finish by showing how to go from the set $\mathcal{S}_{\text {gen }}(A, B)$ of non-local generic separation to the set $\mathcal{S}(A, B)$ of points where local separation of $A$ and $B$ is not possible. We recall that in [AcAnBg, Section 6], we give an algorithm that produces a list $\mathcal{L}_{A, B}$ of subvarieties of $M$ such that $A$ and $B$ are separable if and only if the shadows $A_{W}, B_{W}$ are generically separated for any $W \in \mathcal{L}_{A, B} \cup\{M\}$. Working locally, we get that for any point $p \in M, A$ and $B$ are separable in a neighbourhood of $p$ if and only if for any $W \in \mathcal{L}_{A, B} \cup\{M\}, A_{W}, B_{W}$ are generically separated in a neighbourhood of $p$. Thus we have

$$
\mathcal{S}(A, B)=\mathcal{S}_{\text {gen }}(A, B) \cup\left(\bigcup_{W \in \mathcal{L}_{A, B}} \mathcal{S}_{\text {gen }}\left(A_{W}, B_{W}\right)\right) .
$$

Altogether we have shown

THEOREM 4.1. $\mathcal{S}(A, B)$ is a closed semialgebraic set. 
5. Semialgebraicity of the local basicness locus. Let $S$ be an open semialgebraic subset of $M$. We recall that $S$ is basic open if it can be described as an intersection of strict inequalities, and $S$ is generically basic open if it is basic open up to a proper subvariety $Y$ of $M$. In this section we show that the set $\mathcal{B}(S)$ of points $p \in M$ such that $S$ is not basic in any neighbourhood of $p$ is semialgebraic. Since the proof follows the same pattern as the one given for the local separation locus we only sketch it, pointing out the differences with this case. Similar arguments (with the obvious changes) can be made for the case of closed basic semialgebraic sets.

As above we consider first the generic question, that is the set $B_{\text {gen }}(S)$ of points $p \in M$ such that $S$ is not generically basic in any neighbourhood of $p$. We have the following analogue to Lemma 1.3:

LEMMA 5.1. $S$ is generically basic in a neighbourhood of a closed semialgebraic set $Z$ if and only if $\widetilde{S}$ is basic in $\mathcal{G}_{Z}$.

We will say that the couple $(M, S)$ is (NC) if $M$ is non-singular and the walls of $S$ are non-singular and normal crossings. In [AcBgVe] it is shown that if $(M, S)$ is (NC), then $S$ is not generically basic if and only if either ${\overline{\partial S^{*}}}^{Z} \cap S^{*} \neq \emptyset$ or there is a wall $W$ of $S$ such that the shadow $S_{W}$ of $S$ in $W$ is not generically basic. To get the analogue to Corollary 1.5 from the lemma above, we have to isolate the set $B_{0}(S)$ of points $p$ such that for any neighbourhood $U,{\overline{\partial(S \cap U)^{*}}}^{Z} \cap(S \cap U)^{*} \neq \emptyset$. They are the points in the walls $W$ of $S$ which are in both $\overline{S^{*}}$ and $\operatorname{Adh}_{W}\left(\partial S^{*} \backslash \overline{S^{*}}\right)$. To be more precise, we have

$$
B_{0}(S)=\bigcup_{W}\left(\operatorname{Adh}_{W}\left(W \cap S^{*}\right) \cap \operatorname{Adh}_{W} \operatorname{Int}_{W}\left(W \cap \partial S^{*}\right)\right) .
$$

Thus, we get

Corollary 5.2. Assume that $(M, S)$ is (NC). Then $S$ is generically basic in a neighbourhood of $Z$ if and only if $Z \cap B_{0}(S)=\emptyset$ and for all walls $W$ of $S$ with $Z \cap W \neq \emptyset$ the shadow $S_{W}$ is generically basic in a neighbourhood of $Z \cap W$ in $W$.

P r o of. The condition $Z \cap B_{0}(S)=\emptyset$ means that there is a neighbourhood $U$ of $Z$ such that ${\overline{\left(\partial(S \cap U)^{*}\right)}}^{Z} \cap(S \cap U)^{*}=\emptyset$. Now the result follows from [AcBgVe, Theorem 4.1], taking into account that if $Z \cap W=\emptyset$ then $\mathcal{G}_{Z} \cap \widetilde{W}=\emptyset$.

Theorem 5.3. Assume that $(M, S)$ is $(\mathbf{N C})$. Then the set $B_{\text {gen }}(S)$ is closed semialgebraic.

Proof. It is immediate that $B_{\text {gen }}(S)$ is closed. To see that it is semialgebraic we work by induction on $d=\operatorname{dim} M$. If $d=1$ every semialgebraic set is generically basic, so that $B_{\text {gen }}(S)=\emptyset$ which is trivially semialgebraic.

For higher dimensions we have

$$
B_{\text {gen }}(S)=\overline{B_{0}(S)} \cup\left(\bigcup_{W} B_{\text {gen }}\left(S_{W}\right)\right)
$$

and we are done by induction.

Next we construct the basicness tree for a (NC) couple $(M, S)$ as follows:

1. We start with the single node $(M, S)$.

2. For each node $(N, T)$ with $\operatorname{dim} N>1$ consider $T^{b}=T \backslash B_{0}(T)$. Then, for each wall $W$ of $T^{b}$ we define an edge descending from $(N, T)$ and ending in $\left(W,\left(T^{b}\right)_{W}\right)$. 
Working by induction on the height of the tree, we get

Proposition 5.4. Let $(M, S)$ be (NC) and $Z$ a closed semialgebraic subset of $M$. Then $S$ is generically basic in a neighbourhood of $Z$ if and only if for all nodes $(N, T)$ of the basicness tree $Z \cap B_{0}(T)=\emptyset$.

Finally, using a desingularization morphism and stratifying in the same way as in Sections 3 and 4 for the separation, we get that for any $(M, S)$ the set $B_{\text {gen }}(S)$ is semialgebraic, and since $\mathcal{B}(S)$ can be written as a finite union of $B_{\text {gen }}\left(T_{k}\right)$, cf. [AcBgVe], we get the wanted result:

Theorem 5.5. For any $(M, S)$ the set $\mathcal{B}(S)$ is closed semialgebraic.

6. Non-archimedean real closed ground field. We finish the paper with an example showing that Theorems 4.1 and 5.5 are not true over a non-archimedean real closed ground field $R$. We only deal with the separation problem, the basicness one being analogous. Consider the space $R^{4}$ with coordinates $x, y, z, t$. For any fixed $\varepsilon$, consider in the hyperplane $t=\varepsilon$ the cones with vertex $(0,0,1, \varepsilon)$ over the sets $A_{\varepsilon}$ and $B_{\varepsilon}$ of the picture, placed in the plane $t=\varepsilon, z=0$ (cf. [AcAnBg, Example 5.2], and also [AnBrRz, Example VI.7.10]).

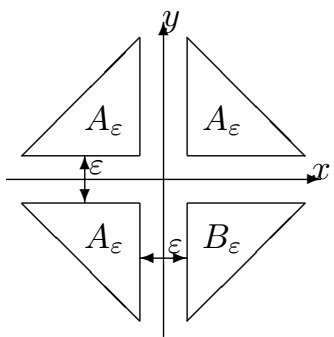

We recall that these sets $A_{\varepsilon}$ and $B_{\varepsilon}$ can be generically separated in the plane $x, y$ if and only if $\varepsilon$ is not infinitesimal (cf. op. cit.).

Finally, let $A$ and $B$ be the collection of all these cones when $0<t<1$. That is,

$A=\left\{(x, y, z, t)=s(0,0,1, t)+(1-s)\left(x_{0}, y_{0}, 0, t\right) \mid\left(x_{0}, y_{0}, 0, t\right) \in A_{t}, 0<s<1,0<t<1\right\}$

$B=\left\{(x, y, z, t)=s(0,0,1, t)+(1-s)\left(x_{0}, y_{0}, 0, t\right) \mid\left(x_{0}, y_{0}, 0, t\right) \in B_{t}, 0<s<1,0<t<1\right\}$

Obviously $A$ and $B$ are open disjoint semialgebraic sets and

$$
\bar{A} \cap \bar{B}=\{(0,0,1, t) \mid 0<t<1\}
$$

so that the set $S(A, B)$ of non-local generic separation is contained in this set. Moreover, for each $\varepsilon$ the sets $A$ and $B$ are locally generically separated at $(0,0,1, \varepsilon)$ if and only if the sets $A_{\varepsilon}$ and $B_{\varepsilon}$ are generically separated in the plane $z=0, t=\varepsilon$, that is, if and only if $\varepsilon$ is not infinitesimal in $R$. Therefore we have

$$
S(A, B)=\{(0,0,1, t) \mid 0<t \text { and } t \text { is infinitesimal in } R\}
$$

which is not semialgebraic. 


\section{References}

[AcAnBg] F. Acquistapace, C. Andradas, F. Broglia, Separation of semialgebraic sets, Preprint, 1996.

[AcBgVe] F. Acquistapace, F. Broglia, M. P. Vélez, Basicness of semialgebraic sets, Geom. Dedicata, to appear.

[AnBrRz] C. Andradas, L. Bröcker, J. M. Ruiz, Constructible Sets in Real Geometry, Ergeb. Math. Grenzgeb. (3) 33, Springer, Berlin, 1996.

[BCR] J. Bochnak, M. Coste, M.-F. Roy, Géométrie algébrique réelle, Ergeb. Math. Grenzgeb. (3) 12, Springer, Berlin, 1987.

[Hk] H. Hironaka, Resolution of singularities of an algebraic variety over a field of characteristic zero, Ann. of Math. (2) 79 (1964), I: 109-203, II: 205-326. 